\title{
Acercamiento metodológico al estudio del poder local: los ayuntamientos de Chiapas (México), siglo XIX
}

María Dolores Palomo Infante

CIESAS Sureste (México) 



\title{
Acercamiento metodológico al estudio del poder local: los ayuntamientos de Chiapas (México), siglo XIX
}

\section{Methodological approach to the study of local power: the town halls of Chiapas (Mexico), 19th century}

\author{
María Dolores Palomo Infante \\ CIESAS Sureste (México) \\ aldonza1717@hotmail.com
}

Fecha de recepción: 14 de diciembre de 2020

Fecha de aceptación: 10 de abril de 2021

\section{Resumen}

El artículo presenta una propuesta metodológica para el análisis del poder local en América Latina en el siglo XIX, particularmente en Chiapas, México. El estudio se centra en los ayuntamientos de pueblos indígenas ladinizados, aquellos en los que la mayor parte de su población era indígena, pero tenían un número importante de vecinos ladinos. Este factor, junto con la trayectoria histórica y el contexto del momento, caracterizó el desarrollo del gobierno local en la región. $\mathrm{El}$ artículo señala algunas preguntas, temas pendientes y perspectivas de análisis de esta institución, teniendo en cuenta, además, que la información documental disponible es escasa. Se analizarán algunos aspectos que caracterizaron los procesos históricos de Chiapas relacionados con esta institución: carácter étnico de la población, consecuencias de la aplicación de los postulados de la constitución de Cádiz -igualdad jurídica, ciudadanía- formas de representación, carácter de los ayuntamientos.

Palabras clave: América Latina; Siglo XIX; Ayuntamientos; Pueblos indígenas; Autoridades indígenas

\footnotetext{
Abstrac

The article presents a methodological proposal for the analysis of local power in Latin America in the 19th century, particularly in Chiapas, Mexico. The study focuses on the ayuntamientos of ladinized indian village, those that most of their
} 
population was indian, but had a significant number of Ladin neighbors. This factor, along with the historical trajectory and context of the moment, characterized the development of local government in the region. The article points to some questions, outstanding topics and perspectives of analysis of this institution, taking into account, moreover, that the documentary information available is scarce. We will analyze some aspects that characterized the historical processes of Chiapas related to this institution: ethnic character of the people, consequences of the application of the postulates of the Constitution of Cadiz - legal equality, citizenship - forms of representation, character of the ayuntamientos.

Keywords: Town councils; Latin America; $19^{\text {th }}$ century; Indian village; Indian authorities.

\section{INTRODUGGIÓN}

El estudio del poder y las instituciones locales puede acercarnos al conocimiento de la cotidianeidad de la población y de otros procesos que se desarrollaron en los pueblos de América Latina. Sin embargo, en algunas regiones, las fuentes de información son limitadas, y en muchos casos inexistentes. Si además se trata de pueblos indígenas nos encontramos con una dificultad adicional para la investigación, ya que la información disponible sobre esta población es escasa, sobre todo en el siglo XIX, cuando la igualdad jurídica decretada pretendió -y en muchos casos consiguió- hacer desaparecer a los indígenas de los pueblos y también de los documentos. Además, como bien sabemos, la mayoría de los documentos no fueron escritos por ellos mismos, sino a través de interlocutores o de otros "escritores" que dejaron la impronta de su visión en sus papeles. Es la útil "ventriloquía", recurso conceptual y metodológico que nos ha legado Andrés Guerrero. ${ }^{1}$

Sin embargo, esta relativa ausencia de información no debe hacernos desistir. Por eso, lo que planteo en este texto es un acercamiento metodológico para el análisis de los ayuntamientos de los pueblos de Chiapas. Más concretamente, me centraré en aquellos pueblos de indios coloniales que tuvieron un proceso de ladinización, por lo que el desarrollo del gobierno local y las relaciones resultantes de la interacción étnica fueron más interesantes y dinámicas.

Chiapas es una de esas regiones en las que desafortunadamente las fuentes de información son pocas, porque los archivos han desaparecido o son difíciles de consultar, por lo que acercarse al conocimiento de esta institución se convierte en un reto. Esto ha provocado una cierta escasez de estudios sobre el gobierno local de los pueblos, sobre todo en aquellos que mantuvieron su población y corporación municipal exclusivamente indígena. Sin embargo, el análisis de los ayuntamientos nos acerca a otros aspectos sociales y políticos de los pueblos. Por ello, para conocerlos es

1 Guerrero (2010) propone el concepto "ventriloquía" para analizar cómo otros personajes hablan por el indio a la hora de presentar sus demandas; son sus interlocutores en un contexto de administración de poblaciones. Es un concepto muy útil a la hora de enfrentarnos con los documentos. 
necesario enmarcarlos en contextos históricos relacionados con el carácter étnico de la población, con la aplicación de los postulados de la constitución de Cádiz -igualdad jurídica, ciudadanía- con el desarrollo de las formas de representación o el carácter de los ayuntamientos, entre otras. Esto nos permite ampliar la búsqueda de información en otros archivos y tipos documentales, más allá de los municipales.

Este trabajo es una propuesta metodológica para el estudio de los gobiernos locales, desarrollada a través del planteamiento de algunas preguntas sobre temas pendientes; además, realizaremos un análisis de las características de esta institución, señalando algunas de las cosas que ya sabemos sobre ella, así como los aspectos que será importante indagar, y explorar de qué forma podemos hacerlo, es decir, diferentes perspectivas de análisis.

También realizaré algunas sugerencias sobre información documental que podemos utilizar para completar los datos de los archivos municipales. El periodo que abarca este estudio parecer muy amplio e inespecífico; sin embargo, el propósito es hacer una propuesta de análisis considerando las características generales que comenzaron a definirse desde finales del XVIII, pero que adquirieron su carácter más nítido a lo largo de la centuria decimonónica. No obstante, es necesario señalar algo obvio: hay que tener en cuenta las transformaciones de la institución en diferentes contextos históricos a lo largo del siglo XIX, con lo que también cambió su significado en cuanto a las relaciones entre Estado, pueblos indígenas y otros grupos sociales.

\section{UN PRIMER ACERCAMIENTO}

Todas nuestras investigaciones rinden tributo a trabajos previos. En el tema de los ayuntamientos, varios autores en México y en América Latina han abordado su análisis desde diferentes enfoques que van desde el institucional, para ver su conformación, integración y la lógica de su establecimiento en cada pueblo, hasta aquellos que reflexionan sobre su dinámica e importancia para los pueblos desde el punto de vista económico, político, social, territorial. También se han realizado análisis sobre su relación con el ámbito judicial; sobre las disputas entre diferentes espacios políticos por el poder, incluyendo las que se produjeron con un carácter interétnico, que por lo general fueron el motor de transformaciones. ${ }^{2}$ También se ha analizado en relación a

2 Sobre los ayuntamientos en México pueden consultarse varios trabajos que contienen los diferentes enfoques y abordajes, y están centrados en distintas regiones. Pueden consultarse los capítulos de los libros: Ortiz Escamilla y Serrano Ortega (2007) que muestran la diversidad del fenómeno municipal a través del análisis en diferentes regiones, centrados principalmente en el periodo de influencia directa de la Constitución gaditana, 1812-1814 y 1820-1824, y el primer federalismo mexicano. También Salinas Sandoval et Al. (2011); Gayol (2012). Para Yucatán, véase Güemez (2005) y Machuca Gallegos (2014). Para Oaxaca, tenemos los trabajos de Mendoza (2011); para las Huastecas, los de Escobar Ohmstede (1997); para el Valle de México, a Marino (2006); para Michoacán podemos consultar a Cortés Máximo (2012); y para Jalisco a Vallejo (2017) Para el análisis de las elecciones y de la ciudadanización de la población, son de obligatoria consulta los trabajos que se contienen 
los cambios y continuidades de la institución con respecto a los cabildos coloniales, el ritmo de transformación a lo largo del tiempo, desde su emergencia con la constitución gaditana y su establecimiento tras la independencia y el nacimiento de los Estados nacionales. En este sentido, otros autores han reflexionado sobre su fortalecimiento o debilidad en comparación con los gobiernos locales coloniales, es decir, en el paso de repúblicas de indios a ayuntamientos constitucionales; ${ }^{3}$ también, qué tan profunda fue la transformación en cuanto al carácter del ejercicio del poder y del gobierno, lo que nos coloca en la discusión sobre la modernidad y su influencia en las sociedades. ${ }^{4}$

Es necesario plantear las diferencias y semejanzas que los gobiernos locales tuvieron en distintas regiones, atendiendo sobre todo a cuestiones económicas, sociales y políticas y según las características étnicas. Es interesante la idea que propone Guarisco (2003), al señalar que la relevancia de los gobiernos locales y de la participación de la población indígena en los procesos políticos dependió en cierta medida de los tipos de sociabilidad coloniales, es decir, de cómo se establecieron las relaciones entre los diferentes grupos de población a lo largo de la colonia, particularmente a finales del siglo XVIII. Estas sociabilidades fueron diferentes en cada región, y dependieron, entre otras cosas, del carácter étnico de la población, que condicionaba las relaciones sociales, por lo que este sería un factor de peso para diferenciar entre los ayuntamientos de cada una de ellas. En este sentido, podemos señalar cierta similitud entre los gobiernos locales de Chiapas y Centroamérica, debido al pasado colonial común y a características étnicas y culturales similares, particularmente con Guatemala, cuya estrecha vinculación llega hasta el presente. Las experiencias de los ayuntamientos en sus pueblos indígenas nos permiten comparar y comprender varios aspectos de los chiapanecos. ${ }^{5}$

Son muchas las lagunas que tenemos sobre el conocimiento de los gobiernos municipales decimonónicos en Chiapas. Los trabajos realizados hasta el momento son escasos, en comparación con otras regiones, por lo que este tema se constituye como un campo fértil para los historiadores de y sobre Chiapas. Ha iniciado tímidamente cierto interés por la historia de esta institución y por los procesos municipales contemporáneos, ${ }^{6}$ pero aún hay muchas cosas sobre las que indagar

en los libros de Annino (1995), Gantús y Salmerón (2019) y de Sábato (1999). Para estudios sobre otros países: Rodas (1995 y 1997), Avendaño (1997), Barrios (1998), Alda (2000), Esquit (2002) para Guatemala; Herrera Mena (2013) para El Salvador; Palomeque Torres, 1992 y 2000 y Borrero Vega (2014), para Ecuador. Sobre Perú, Peralta (1991) y Thurner (1996).

3 Cortés Máximo (2012). En este debate destacan los trabajos de Guerra (1994 y 1995), Annino (1995 y 2003). En la misma línea podemos encontrar un artículo de Buve (2007).

4 Ver Guarisco (2019).

5 Para bibliografía, ver nota núm. 2.

6 Recientemente ha sido publicado un libro con una recopilación de trabajos sobre la historia de esta institución en Chiapas, analizada desde diferentes campos (Ortiz Herrera, 2018a). Otros autores que han abordado el tema son: Ortiz Herrera (2003), Vázquez Olivera (2010), Palomo (2009b y 2015), Gutiérrez y Parrilla (2018). Desde la antropología, hay algunos trabajos sobre el municipalismo contemporáneo: Rodríguez (2003), Burguete Cal y Mayor, (2011 y 2016), entre otros. 
para dar una visión más completa y compleja sobre los ayuntamientos en este estado. Por eso la propuesta metodológica que presento se centrará en ellos.

\section{HACIA UN ANÁLISIS CONGEPTUAL}

Al hablar del siglo XIX, necesariamente tenemos que hacer referencia a los conceptos que surgieron desde la ideología liberal decimonónica, y que ha usado la historiografía para el análisis de este periodo histórico. Es importante que en las investigaciones sobre el siglo XIX se tengan presentes estos conceptos, para discutirlos y analizarlos en su relación con el contexto histórico local y regional, ya que la mayor parte de los procesos estuvieron determinados por ellos. Me refiero a: Liberalismo, Estado, Nación, Ciudadanía, Vecindad, Igualdad jurídica y su relación con el poder, el gobierno y la autoridad en el tema que ahora nos ocupa. No hay consenso sobre lo que implicaron estos conceptos para todas las regiones de América Latina, e incluso para Europa, porque además no en todas las regiones tuvieron el mismo efecto. La regionalización se impone sobre la globalización, por lo tanto, el análisis de su aplicación en contextos regionales específicos puede aportar mucho para desentramar su significado conceptual.

Hay una amplia bibliografía sobre el liberalismo desde diferentes enfoques: teóricos, políticos, filosóficos...; $;^{7}$ pero lo que más interesa es valorar la responsabilidad que tuvo en cada uno de los ámbitos en los que se produjeron transformaciones con respecto al Antiguo Régimen (Escobar et Al, 2015), así como sus efectos sobre las instituciones y los actores sociales (Annino, 2002). Esto nos permite cuestionarnos si el liberalismo produjo cambios profundos en su camino hacia la Modernidad; o en qué condiciones y circunstancias lo hizo. En este sentido, Guerra (1993) propone diferentes ritmos de cambios, a lo que habría que sumar las particularidades locales y regionales que imprimieron un tiempo particular en cada caso. ${ }^{8}$ Ahora bien, ser liberal, ¿significó lo mismo en todas las partes?, ¿por qué los identificamos ahora como liberales o conservadores? En Chiapas, se ha establecido una bipolaridad histórica entre liberales -tierras bajas- y conservadores -tierras altas- que todos seguimos sin discusión y que, desde muchos puntos de vista, no ha sido suficientemente discutida. Es importante la reflexión que ha realizado sobre este tema Torres Freyermuth (2017), pero debemos seguir profundizando al respecto.

Acerca del Estado y la Nación son conceptos muy amplios, y no es el propósito de este trabajo una discusión sobre ambos. ${ }^{9}$ Lo que sí nos interesa es ver cómo se pasó de un "Estado" colonial, a uno independiente, nacional, republicano, así como

7 Véase Galante (2004); Ávila (2007) para un balance historiográfico.

8 Véase Guerra (1993), Alda (2000), Marino (2006) entre otros autores.

9 Lempérière (2007) realiza una interesante reflexión sobre la historiografía del Estado. Sobre la nación, Escobar (2010). También Escobar et Al. (2010) 
su proceso de consolidación y fortalecimiento. Este es un aspecto importante desde el punto de vista historiográfico e histórico, pues trajo aparejado cambios y permanencias en varios ámbitos de la vida de los pueblos y en la relación entre ambos. Para Chiapas, el clásico trabajo de Benjamin (1990) sigue siendo casi el único que nos acerca a una reflexión sobre este tema. Propone que a lo largo del XIX, el Estado fue débil en Chiapas y sólo a finales de siglo logró fortalecerse para modernizar esta región y crear un nuevo orden político que la integrara a la república. Otras investigaciones sobre diferentes temas han abordado algunos aspectos relacionados con él, pero es necesario realizar aportaciones a la luz de nuevas fuentes documentales y nuevos enfoques. Sin duda, esta reflexión es necesaria para el análisis de las instituciones de gobierno local.

En el siglo XIX comenzaron a gestarse las naciones latinoamericanas. Es necesario reflexionar sobre los criterios que los nuevos Estados estaban contemplando para definir su Nación. Se pretendía que una de sus características fuera la homogenización (Quijada, 2000), por lo que se decretó la igualdad jurídica, que tuvo una relevancia significativa en países en los que la diversidad estaba presente, como Guatemala o México. En este último, por ejemplo, la homogeneidad fue importante cuando el nacionalismo y la nación se convirtieron en el centro de las políticas del Estado mexicano. Y como cada nuevo país tenía particularidades regionales muy acentuadas, es importante deslindar lo que cada región aportó a ese concepto de Nación.

Lo mismo sucede con el término Ciudadanía. ¿Qué es y qué se entendió por ciudadanía en el siglo XIX? Con ella y con la igualdad jurídica que se decretaron desde la Constitución de Cádiz, se pretendió homogeneizar a todos los habitantes de una nación, pero ¿significó realmente una igualdad de los individuos; se daban las condiciones para ello? ¿qué cualidades debían tener los ciudadanos? Si tenemos en cuenta que para ser ciudadano se necesitaba saber leer y escribir, tener bienes y un oficio reconocido, es obvio pensar que no todos los habitantes de la nación cumplían con estos requisitos, por lo tanto ¿dónde se ubicaban aquellos individuos que no las tenían y que, por esa razón, eran excluidos de los cargos importantes del ejercicio del poder y del gobierno? ¿no podremos interpretar la ciudadanía como una reelaboración delas formas de dominación?. ${ }^{10} \mathrm{Pero}$, por otra parte, también podemos hacer preguntas en otro sentido: ¿fueron sólo estos criterios ciudadanos los que determinaron la representación? O ¿había otros caminos para desempeñar los cargos de gobierno? O ¿es posible que hubiera otras formas de "ser ciudadanos"? Autores de diferentes regiones como los Andes, Guatemala o México se cuestionan en este sentido (Irurozqui, 2004; Esquit 2002, p. 52; Palomo, 2018; Acevedo y López, 2012).

10 En Guarisco (2019) se analiza cómo la ciudadanía en cada uno de los periodos después de la independencia hasta en la primera mitad del siglo XIX ampliaba o reducían las posibilidades que los indígenas tenían para ocupar cargos en los gobiernos locales. Alda (2000) por su parte resalta la controversia surgida en Guatemala entre dotar de igualdad al indio y a su vez mantener su tutela. 
En cuanto al concepto de vecindad, podemos preguntarnos ¿quiénes eran los vecinos? Jurídicamente estaba claro, pero ¿cómo se aplicó en la realidad? ¿Era realmente el sustento o complemento de la ciudadanía? ¿cómo se establecía la vecindad? ¿qué pasaba con quiénes no estaban avecindados en ningún pueblo? ¿entraban todos en la categoría de "vagos"? Estas preguntas nos remiten realmente a la discusión sobre el tipo de Nación que subyacía en las mentes y en los proyectos de los políticos decimonónicos. Pues bien, nuevamente aquí hay mucho que reflexionar, ya que todos estos conceptos están en estrecha relación con el desarrollo de los ayuntamientos y el poder local.

\section{LOS PUEBLOS Y SUS HABITANTES}

Como señalaba más arriba, el desarrollo de los ayuntamientos estuvo en estrecha relación con las circunstancias locales y el contexto histórico en el que se desarrollaron; su vida estuvo inevitablemente unida a la de los pueblos, por lo que es necesario describir a éstos últimos y a sus habitantes para entender y contextualizar la trayectoria de esta institución.

Un primer aspecto que considero importante es de índole demográfica. Una característica de los pueblos chiapanecos - que tiene una especial relevancia para el análisis de los gobiernos municipales-es que algunos eran habitados por indígenas en su totalidad, mientras que otros desde muy temprano vivieron un proceso de ladinización, como Ocosingo, Teopisca y Comitán. ${ }^{11}$ Entre otras razones esto se debió, fundamentalmente, a los movimientos de población que se realizaron por diferentes motivos -económicos, fundamentalmente-; me refiero a la llegada de mestizos y/o españoles a los pueblos indígenas procedentes de otros lugares. Sabemos que algunos se ladinizaron desde finales del siglo XVIII. Un ejemplo es el de Teopisca en el que vecinos ladinos de la cercana Ciudad Real de Chiapas adquirieron algunas tierras desde el siglo XVIII. Esto provocó la ladinización de la cabecera, donde el 50\% de su población era ladina a mediados de la centuria decimonónica (Barrera Aguilera, 2017, p. 81). Este hecho se vio favorecido por los cambios legales que eliminaron la prohibición colonial que impedía a los españoles, mestizos y castas vivir en los pueblos de indios.

Ahora bien, también podemos entender la ladinización desde un enfoque metodológico que considere la cuestión cultural de los pueblos; es decir, teniendo en cuenta la forma en que los contemporáneos definían o calificaban a los "ladinos". Es un concepto que fue cambiando a lo largo del tiempo. En 1748, fray Agustín de Arévalo, cura doctrinero de Teopisca y sus anexos, consideraba a los ladinos como

11 Para Guatemala, Alda (2000) y Esquit (2002) han mostrado procesos semejantes en sus investigaciones sobre el Occidente del país. 
"mixtos de españoles e indios y mulatos" ${ }^{2}$. Más tarde, en el mismo pueblo, en un informe parroquial elaborado alrededor de $1 \wedge 10$ por el presbítero Agustín Maza, se separaba a los mestizos ( 1 r. "almas") de los individuos que se integraban en "las demás castas" ( $0 \wedge$ "almas")". Mientras, el "Informe rendido por la Sociedad Económica de Ciudad Real", de 1819, reportó datos muy parecidos al anterior, pero incluyó a "las demás castas" en el grupo de los ladinos (177 en total). Por último, el ayuntamiento ladino de Ocosingo, en 1879, señalaba que una parte importante de su población estaba compuesta de indígenas y ladinos pobres..."14

La ladinidad o indianidad de cada pueblo influyó en las formas del ejercicio del gobierno y las relaciones socio políticas entre sus habitantes. Por lo tanto, un tema pendiente en la agenda de los estudios de los poderes locales en Chiapas es discutir estos conceptos y analizar los procesos demográficos desde finales del siglo XVIII y a lo largo del siglo XIX, para conocer las características poblacionales de los pueblos. ${ }^{15} \mathrm{El}$ potencial económico de cada uno de ellos fue sin duda una de las razones que impulsó su ladinización; a su vez, estos adquirieron importancia política, al convertirse en cabeceras de partido o departamento. Con un mayor número de ladinos en los pueblos y la legislación que favorecía la igualdad jurídica, se dieron todas las circunstancias para que los indígenas fueran desplazados de los cargos de la institución municipal. A esto se sumó la supuesta mejor preparación y "calidad" social de los ladinos; saber leer y escribir era imprescindible ahora para ocupar un cargo concejil. Pero también lo definía tener una buena posición económica, según los criterios de ciudadanía. En este punto debemos señalar que no todos los ladinos tenían una situación económica sólida y estable; algunos incluso estaban en la misma situación económica que los indios.

En esta coyuntura, los ladinos reclamaron derechos políticos en los pueblos, lo que les permitiría defender mejor sus intereses, sobre todo económicos. Esta situación fue favorable para que, en algunos pueblos, comenzaran a ocupar cargos concejiles lo que tuvo repercusiones en el ámbito del gobierno y de la justicia.

12 Archivo Histórico Diocesano (en adelante AHD). Asuntos parroquiales. Teopisca. IV.D.3. 1748 .

13 AHD. Asuntos parroquiales de Teopisca (IV-D-4. 1810-1833) Padrones eclesiásticos. 1814.

14 Archivo Histórico del Estado (en adelante AHE), Fondo Castañón y Gamboa (en adelante FCG). Expediente 573. Actas de las sesiones del ayuntamiento de Ocosingo y otros documentos importantes. 1879 .

15 Hay algunos trabajos que nos marcan el camino: Obara (2010); Barrera Aguilera, (2017); Palomo (2020). La lengua, la fiscalidad y la clasificación de poblaciones por el Estado fueron, entre otros, algunos de los factores que definieron la ladinización. 


\section{ESTABLECIMIENTO DE LOS AYUNTAMIENTOS EN CHIAPAS}

El 19 de marzo de 1812 con la promulgación de Constitución Política de la Monarquía española, comenzó a definirse el desarrollo del ayuntamiento constitucional, como institución administrativa para el gobierno interior de los pueblos. En ese mismo acto se legalizó la desaparición del cabildo colonial que por trescientos años había regido la vida política de los pueblos del Nuevo Mundo, aunque no su desaparición total. Este fue uno de los primeros grandes cambios del liberalismo.

En Chiapas, los ayuntamientos comenzaron a funcionar en la década de 1820. No hemos encontrado información sobre su establecimiento durante el primer periodo gaditano, excepto un documento sobre el que funcionaba en San Cristóbal de las Casas, en $1814 .{ }^{16}$ Vázquez Olivera (2010, p. 80) señala la relevancia que tuvieron para las elites chiapanecas, que se valieron de ellos para afianzar su presencia en diferentes regiones. Ortiz Herrera (2003, p. 194) dice que los primeros ayuntamientos constitucionales comenzaron a funcionar en Chiapas en 1826, con la promulgación de la constitución estatal del mismo año. Según la documentación consultada, tenemos constancia de su existencia antes de 1826 en: San Miguel Pinola (1824), Teopisca (1825), ${ }^{17}$ Soyatitán (1825) y Santa Cruz Sosocoltenango (1826) ${ }^{18}$ así como en otros pueblos ${ }^{19}$ que, juntos con los de las cabeceras de partido, fueron instalados durante el segundo periodo de la constitución gaditana y el Primer Imperio. La falta de documentación y lo convulso de esos años no nos permiten conocer más acerca de ellos, sin embargo, estuvieron presentes en los momentos trascendentales de la historia del estado, como en la decisión de integrarse a México. ${ }^{20}$ En 1827 había 40 ayuntamientos constitucionales: uno en Ciudad Real, capital del estado; 15 ladinizados y el resto "son compuestos de puros indígenas; y solo en la forma son constitucionales, pu[es] se hallan incapaces de llenar las funciones de su encargo. Firma Ygnacio Velasco."21

16 La referencia está en un documento que se encuentra en el Archivo Histórico Diocesano de San Cristóbal de Las Casas. Agradezco a Amanda Úrsula Torres Freyermuth haberme proporcionado la información.

17 AHE, Fondo Archivo Histórico de Comitán, Presidencia Municipal, 1826.

18 AHE, Fondo Archivo Histórico de Comitán, Presidencia Municipal, 1826.

19 En el documento del Archivo General de Centroamérica, Guatemala C.A (en adelante AGCA) Guatemala A1-57318-6932 (1821) hay una lista de ayuntamientos constitucionales que existían en Chiapas en 1821: Ciudad Real, Zinacantán, Chamula, Comitán, San Bartolomé, Tuxtla, Chiapa, Tonalá, Tapachula, Huixtán, Ocosingo, Palenque, Ixtacomitán, Pueblo Nuevo Pichucalco, San Andrés y Simojovel. Agradezco a la Dra. Ana María Parrilla Albuerne haberme proporcionado el citado documento.

20 Véase Vázquez Olivera (2010)

21 Instituto Nacional de Antropología e Historia (2007). 
El 30 de noviembre 1825, se publicó la Ley reglamentaria del ayuntamiento, ${ }^{22}$ que tuvo una larga vigencia a lo largo del siglo XIX. La legislación normaba el funcionamiento del cuerpo municipal, principalmente en cuanto a las condiciones que debían cumplir los pueblos para fundar una corporación municipal -por lo general, el número de habitantes-, y la dinámica interna de estas. Sin embargo, la práctica diaria podía modificar las normas. La constitución de Chiapas de 1826, en el artículo 75 señalaba que "Para el gobierno interior de los pueblos habrá ayuntamientos elegidos popularmente en todos los que tengan el número de mil almas a lo menos; o aunque sea menor su población, si así lo exigen sus circunstancias". ${ }^{23}$ Sabemos que una de sus principales funciones fue la impartición de justicia, razón suficiente para que se fundaran en pueblos que no alcanzaban el número de habitantes necesarios.

El establecimiento de ayuntamientos a lo largo del siglo XIX estuvo determinado por los cambios en el carácter del gobierno de la república. Durante los periodos federalistas, se fortalecía su posición en los pueblos y su autonomía con respecto al Estado, mientras que con el régimen centralista era todo lo contrario: se suprimían los ayuntamientos o se reducían al máximo en sus funciones en favor de autoridades controladas por el gobierno central, como los jueces de paz -que tenían funciones de justicia y también tendrían "la dirección de los ramos municipales" en algunos momentos ${ }^{24}$ - o los comisarios. ${ }^{25}$ Los años centrales del siglo XIX - de 1836 a 1856fueron de constante cambio para la vida de los gobiernos locales.

En los pueblos de indios coloniales, el cabildo estaba integrado por autoridades que representaban tanto a las cabeceras como a las parcialidades. Esto fue importante en Chiapas debido a la forma en que se crearon los pueblos de indios, a través de la reunión de poblaciones que anteriormente no había tenido ninguna relación, y eran independientes desde el punto de vista político. Algunos de estos poblados congregados se conformaron como parcialidades del nuevo pueblo de indios. En estas circunstancias, era importante tener representación en el cabildo a través de sus justicias para no perder la capacidad de decidir. Así, en el acto de posesión de encomienda de la parcialidad de Tepiencoapa, en 1613, se hallaban presentes el alcalde de la parcialidad, los principales y el cabeza de calpul. ${ }^{26}$ Con el surgimiento

22 AHE, Fondo Archivo Histórico de Comitán, Presidencia Municipal, 1825.

23 Constitución Política del estado de Chiapas, 1826.

24 Decreto de gobierno. Se designan los lugares en que debe haber ayuntamiento, 20 de mayo de 1853, en Dublán y Lozano (1876). En 1837 se dispuso que tendrían carga concejil. Reglamento provisional para el gobierno interior de los Departamentos, de 20 de marzo de 1837. Dublán y Lozano (1876)

25 Estaba regulado por la suprema circular de 5 de octubre de 1853. "Circular de 11 de febrero de 1854". AHE-FCG, expediente 138. Cuaderno copiador de la subprefectura y del juzgado de paz de Ocosingo, 1853-1854.

26 Archivo General de Indias (en adelante AGI). Audiencia de Guatemala 97, N. 31, 28 de marzo de 1615. Confirmación de encomienda de Iztacomitlan, Bitilan, Oiexcuatlan, Ocotitlan, Tepiencoapa, y Zitalazitaclan en Chiapa a Pedro de Eizaguirre. 
de los ayuntamientos constitucionales, las parcialidades disminuyeron su presencia y algunos pueblos perdieron su "derecho" a autogobernarse.

En la época colonial, en varias regiones, se habían producido segregaciones de los pueblos sujetos para convertirse en cabeceras (Escobar, 1996). Pero en el siglo XIX, posterior a la constitución de Cádiz, y para el caso de Chiapas, la creación de nuevos municipios no siguió ese proceso de escisión. Por lo general, los mismos pueblos de indios coloniales fueron los que a lo largo del siglo XIX fueron recuperando el derecho a tener ayuntamiento. Otros nuevos se fueron creando, como por ejemplo Las Margaritas (1871), ${ }^{27}$ en el departamento de Comitán; y Pueblo Juárez $(1861)^{28}$, en el de Pichucalco. Según Mendoza, en Oaxaca se llegaron a unir varios pueblos sujetos para formar un municipio, pues de forma independiente no podían hacerlo debido a su población (Mendoza, 2011, p. 389).

Es interesante en este aspecto preguntarnos cómo fue cambiando la fisonomía político territorial del estado a lo largo del siglo XIX, analizar las circunstancias en cada caso, y el efecto que tuvo el ayuntamiento y las políticas liberales sobre las jurisdicciones territoriales. ¿Qué situación determinaba la calidad de "cabecera" y cómo se decidía el número de pueblos dependientes de la misma? ¿qué circunstancias determinaban en la realidad, la creación de un nuevo municipio? ¿el número de habitantes, según marcaban las leyes?; y, por último, también hay que preguntarse qué pasaba en los pueblos que habían perdido o no tenían ayuntamiento, ¿cómo se gobernaban, como era su "poder local"? Legalmente, se nombraron alcaldes auxiliares, pero también continuaron teniendo autoridades propias, comunitarias que, contaban con una gran legitimidad entre los indígenas. Es necesario profundizar en esto.

\section{CARACTERÍSTICA DE LOS AYUNTAMIENTOS EN CHIAPAS}

Los ayuntamientos, como todas las instituciones, se transformaron a lo largo del tiempo; por lo tanto, esta es una de las primeras cuestiones que debemos tener en cuenta a la hora de su análisis: su ubicación en el contexto histórico.

Las primeras características de esta institución se las dio la constitución de Cádiz; aquí ha surgido un interesante debate sobre las diferencias entre el cabildo colonial y el ayuntamiento constitucional; y también sobre la importancia que tuvieron frente al Estado; debate que tiene en Guerra (1994 y 1995) y Annino (1994 y 2003) a dos de sus exponentes.

Es innegable la influencia que tuvo este texto-hecho histórico en la historia de América. Entre otras cosas, en ella se establecía el principio de igualdad jurídica de todos los habitantes de América, que estuvo en la base de la ideología liberal

27 AHE-FCG, expediente 436.

28 AHE-FCG, expediente 245. 
decimonónica, y que tiene sus antecedentes en la época de la Ilustración de finales del siglo XVIII. Además, la constitución reguló el acceso de los individuos a los órganos de poder local a través del sistema de representación basado en la ciudadanía. Si tenemos en cuenta ambos criterios, es decir, la igualdad jurídica y las formas de acceso a los órganos de poder local, y a ello añadimos la ladinización, podemos inferir la enorme trascendencia que la constitución tuvo en los pueblos del territorio chiapaneco. Ahora, con la igualdad jurídica, podríamos pensar que era una coyuntura más favorable para los indígenas, aunque la realidad no siempre fue así, por lo que debemos analizar los matices.

Durante la colonia, estaba prohibido que los ladinos ocuparan cargos de cabildo en los pueblos indígenas. Sin embargo, también se abrió la posibilidad de su acceso a los puestos municipales. Surgió con ello lo que, para Guatemala, Barrios (1998) denomina alcaldía mixta. Esquit (2002, p. 50) señala que, en un primer momento posterior a la independencia, las leyes guatemaltecas "incluyeron a indígenas y ladinos en las municipalidades, conformando las llamadas "municipalidades mixtas", pues el Estado pretendía que ambos grupos participaran en la conducción de la vida" de los pueblos, aunque después ya no se hizo esta especificación.

La legislación mexicana y la chiapaneca no aluden a esta figura de gobierno, en consonancia con la idea de igualdad jurídica; sin embargo, algunos datos que disponemos -escasos, hay que decir- parecen confirmar también la existencia de la municipalidad mixta, aunque no estuviera expresamente así reconocida en las leyes. En 1835, un acta de elección de Ocosingo dice: “....acto continuo, siendo los ciudadanos José Alejo Hidalgo, Eugenio Estrada y Francisco Burguete en el salón de la casa consistorial de esta villa cuyo número de electores son tres por haberse exceptuado los de la municipalidad indígena". ${ }^{29}$ No eran dos municipalidades funcionando separadamente; más bien, parece una municipalidad mixta, compuesta de ladinos e indígenas. Esto sería posible, seguramente, por esos ajustes locales que la realidad tenía que hacer ante la legislación.

¿Qué implicó para indígenas y ladinos compartir el ejercicio de la autoridad? Un ejemplo: el ayuntamiento de Ocosingo, por lo menos entre 1830 y 1834 -que es de cuando tengo información clara- estaba compuesto por regidores ladinos e indígenas. En una reunión de cabildo del 19 de junio de 1831, la parte indígena del pueblo se opuso a que se aprobara un asunto relacionado con la fijación del ejido y la venta de un terreno. En el acta se expresa que los regidores indígenas "salvan su voto adhiriéndose a las ideas de los demás indígenas". ${ }^{30}$ Este ejemplo es uno en el que podemos observar una dinámica conflictiva de esta institución. Para varios casos, los documentos municipales no dan mucha información al respecto; pero como esta convivencia produjo momentos de conflicto, buscar en los archivos judiciales pueden ser una buena opción.

29 AHE-FCG, Expediente 37. Actas de las sesiones ordinarias y extraordinarias. Ocosingo.

30 AHE-FCG, Expediente 23. 
Con la ladinización los resultados fueron dispares. Güemez (2005) señala que, en regiones periféricas de mayoría indígena, los blanco-mestizos desplazaron a los primeros en el ayuntamiento constitucional. Ahora bien, podemos realizar el análisis desde otra perspectiva. En 1847 se decretó en Chiapas la distinción entre los ayuntamientos de los pueblos ladinizados -aunque no los llama así la ley-y aquellos que había en los pueblos de indígenas; estos últimos se "renovarán anualmente conforme a las costumbres recibidas en ellos los que tendrán también gobernadores que serán vitalicios, elegidos por sus principales y no podrán ser removidos del destino si no es por causa legalmente comprobada" ${ }^{31}$ Prácticamente, se está reconociendo la existencia de gobiernos indígenas o estructuras indias de poder, lo que nos permitiría hacer una distinción entre los ayuntamientos constitucionales versus las autoridades tradicionales, que en definitiva son diferentes formas de ejercer el poder. También Güemez (2005, pp. 22-21) analiza distintas formas de pervivencia de los cabildos indígenas en el régimen constitucional.

Lo anterior nos invita a pensar en la relación entre los ayuntamientos y los sistemas de cargos, jerarquías cívico-religiosas, o autoridades tradicionales, instituciones que tienen una estrecha vinculación en la actualidad, que puede ser cercana y de colaboración o de franco enfrentamiento. Relacionadas con estas jerarquías se encuentran también las cofradías que, junto con el cabildo, ocuparon un lugar importante para los pueblos de indios durante la colonia, hasta finales del XIX (MacLeod, 1983; Palomo, 2009a.) Estas asociaciones religiosas eran un espacio en el que los indígenas podían reunirse para tratar asuntos de la comunidad fuera de la mirada de las autoridades civiles y de los religiosos. Por ello, cuando los ladinos llegaron a las instituciones de gobierno, las reuniones de las cofradías continuaron siendo el espacio para tratar asuntos de carácter civil, a través de los principales de cada pueblo. Chiapas es un estado en el que el peso de las autoridades tradicionales o jerarquías cívico-religiosas ha sido muy fuerte desde el siglo XIX, y han sido abordados por la antropología a partir de mediados del siglo XX; sin embargo, no desde el punto de vista histórico. ${ }^{32}$

Para analizar el funcionamiento real de los gobiernos municipales, nada mejor que remitirse a sus actas, donde se refleja la vida diaria de esta institución y sus problemáticas. Otros documentos municipales que contienen información son los libros que registraban la actividad económica, o las actas de conciliación, entre otros. ${ }^{33}$ En ellas, y en otros documentos, sobre todo judiciales, podemos conocer

31 AHE-FCG, Expediente 084. Decretos del gobierno del estado y federal, así como diversas comunicaciones de la prefectura del oeste con sede en Tuxtla. 1846, 1847.

32 Cancian (1976), Cámara Barbachano (1966) y otros antropólogos que trabajaron en Chiapas a partir de la década de los cuarenta del siglo XX iniciaron los estudios sobre esta institución. Posteriormente, ha sido un tema recurrente de los estudios antropológicos de finales del siglo. Rus y Wasserstrom (1980) hacen un análisis sobre su surgimiento.

33 Tanto las actas de sesiones de cabildo como las de conciliación se localizan en los archivos municipales, pues en los ayuntamientos se ubicaban los juzgados de conciliación, como veremos 
la cotidianeidad de la institución, los principales asuntos municipales, así como el clima social que predominaba en cada momento.

Nuevamente aquí tenemos que hacer una distinción entre la legislación y la realidad diaria. Así, en la primera podemos conocer cuáles eran las funciones de cada uno de los cargos; pero debemos preguntarnos, por ejemplo, sobre las implicaciones que tenía el hecho de la calidad étnica de las autoridades municipales en los pueblos ladinizados: ¿cómo se repartieron las funciones y los cargos? Cuando se distribuían las regidurías entre ladinos e indígenas ¿desempeñaban las mismas funciones los regidores ladinos y los indígenas? ¿no será que estos últimos se convertían en intermediarios entre su población y las instancias de poder, además de recaudadores de impuestos? Reflexionar sobre ello nos coloca en la base de las relaciones políticas y sociales en los pueblos ladinizados.

\section{COMPOSICIÓN DE LOS GOBIERNOS MUNICIPALES}

La legislación marcaba cuántos miembros debía tener cada ayuntamiento, quiénes podían ser electos, quiénes electores, así como los mecanismos de las elecciones. Esto estaba relacionado con la ciudadanía y las formas de representación que surgieron paralelamente a la soberanía popular, que ponía en manos del pueblo la dirección política de la república. En teoría, esto otorgaba a los indígenas buenas condiciones para participar en los gobiernos, lo que para la apreciación de las élites era inadmisible. Si la ley había igualado a todos los individuos de la nación, era importante limitar a través de las restricciones que imponía la ciudadanía la posibilidad de que toda la población, particularmente la indígena, pudiera decidir sobre el destino político.

Si la ciudadanía era restrictiva, en la práctica se deberían adoptar otros criterios que no fueran los ciudadanos -en cuanto a los electores y elegibles- para que los indígenas participaran en los procesos de representación. Debemos preguntarnos si lo anterior se debía a la "necesidad" de representación de todos los grupos de población que componían los pueblos, particularmente en los ladinizados. O si la intención era conservar ciertas prácticas tradicionales indígenas. Guarisco (2019) señala que al menos hasta la primera mitad del siglo XIX el tipo de elección recordó

más adelante. Desafortunadamente, la situación de estos repositorios en el estado de Chiapas es muy mala, por lo que la existencia de estas actas no es sistemática. Particularmente, he trabajado en el Archivo Histórico del Estado, en el Fondo Castañón y Gamboa donde se encuentra la información del ayuntamiento de Ocosingo -actas de sesiones, de conciliación y juicios verbales, junto con otro tipo de documentos municipales y judiciales-, así como expedientes del departamento. También hay actas municipales y de conciliación en el Archivo Histórico de La Trinitaria, aunque contiene poca información del siglo XIX; en el Archivo Histórico de Comitán, en el que hay principalmente información judicial. Por último, los expedientes que contiene el Archivo Municipal de San Cristóbal de Las Casas inician a partir de 1863, por el incendio que sufrió el archivo municipal en ese año. También están disponibles los archivos municipales de Tuxtla Gutiérrez, Chiapa de Corzo y Tapachula con información del siglo XIX. 
en cierto modo un sistema corporativo que prevaleció durante todo este tiempo y que impidió que las instituciones se basaran en el individuo, "premisa insoslayable de la modernidad". Esto es particularmente importante, porque del análisis de quiénes formaban parte de los ayuntamientos, su "calidad" $y$ forma de acceso podemos obtener información sobre cómo se desarrollaron las relaciones políticas en cada momento.

El caso de Ocosingo muestra el procedimiento electoral. La ley no hacía explícita la forma de representatividad étnica en estos procesos. En este pueblo, que tenía una municipalidad mixta, los electores eran tanto ladinos como indígenas, pero no en partes proporcionales al número de población, sino al de cargos de cada "calidad". En la década de los treinta, el número de electores de Ocosingo era cinco. En 1835, no participaron en la elección los dos electores de la municipalidad indígena, con lo cual solo lo hicieron los tres electores ladinos. Ese año eligieron un alcalde, un síndico y seis regidores. Mientras, en 1836, participaron los cinco electores, eligiéndose en este caso como cargos concejiles dos alcaldes, un síndico, seis regidores ladinos y cuatro indígenas. Todos se eligieron en el mismo acto, pero parece que cada grupo de electores elegía a sus autoridades. ${ }^{34}$

Las reglas de participación permitieron la entrada de nuevos grupos sociales en el juego político de los pueblos, que hasta entonces habían tenido cerrada la puerta. Es importante saber a quiénes representan esos grupos sociales, para poder dibujar el entramado de redes políticas y sociales que se entretejieron en cada momento, alrededor del juego de las elecciones..$^{35} \mathrm{~A}$ su vez, los cargos concejiles fueron conformando élites políticas y sociales. Si analizamos los grupos o familias, tanto indígenas como ladinas, que aprovecharon la dinámica de los ayuntamientos para posicionarse en los espacios de poder de los pueblos, podremos observar el mosaico de relaciones sociales y económicas que conformaba este juego político.

Es necesario señalar que los cargos concejiles, a veces podían ser una carga para quienes los ejercían, como se muestra en las solicitudes de licencia. Por lo tanto, podemos preguntarnos ¿qué ventajas tenía ocupar el cargo, si no era la posibilidad de defender los intereses de los grupos de población a los que representaban?

\section{REGURSOS EGONÓMICOS DE LOS AYUNTAMIENTOS}

Los ayuntamientos necesitaban recursos económicos para desempeñar adecuadamente sus funciones. Los principales provenían de los propios y arbitrios. En la documentación existente, esta información está más o menos clara. No lo está tanto las implicaciones que el control y la existencia de dichos recursos tuvieron socialmente.

34 AHE-FCG, Expediente 37. Actas de las sesiones ordinarias y extraordinarias. Ocosingo. 1835.

35 Ver Ortiz Herrera (2018b). 
Un ejemplo: una de las principales fuentes de ingresos de las instituciones de gobierno provenía del cobro de impuestos (arbitrios). Por lo tanto, analizar la provisión de recursos de los ayuntamientos está en estrecha relación con la fiscalidad. Afortunadamente, ya contamos para Chiapas con varios trabajos sobre fiscalidad municipal (Parrilla, 2018; Sánchez Rafael, 2018), pero en lo que debemos profundizar es encómo afectóa la población indígenaestereparto, tanto deingresos comodeegresos.

Un recurso que causó controversia fue la tierra, que entraba dentro de la categoría de los propios, y como tal fue controlada por los ayuntamientos; pero ¿qué recursos se integraron a los propios o fueron gestionados por la corporación municipal? ¿los bienes comunales? Por la documentación generada por la desamortización, sabemos que así fue; el argumento de los denuncios de terrenos realizados por ladinos, contra los que protestaron los indígenas, tenían detrás la gestión que previamente habían realizado los ayuntamientos, lo que creó confusión entre los bienes municipales y los comunales (Palomo, 2007).

Nuevamente en este tema, tenemos que hacer una diferencia entre pueblos indígenas y pueblos ladinizados. A lo largo del XIX, sobre la base de la legislación agraria, una parte importante de las tierras de los pueblos indígenas fue privatizada, lo que provocó conflictos tanto internos como externos. En este sentido, los ayuntamientos indígenas lucharon por la defensa de las tierras, tanto las comunitarias como las de las cofradías, con todos los recursos que tenían a su alcance, muchas veces incluso aprovechando resquicios legales (Palomo, 2007). Mas abajo veremos un ejemplo. Sin embargo, en los pueblos con ayuntamientos ladinizados, la defensa de estos recursos no fue tan evidente ¿qué consecuencias tuvo esta situación para los pueblos indígenas? ¿Cómo se desarrollaron los conflictos por los recursos naturales? ¿cuáles eran las razones por las que se emprendieron los pleitos? ¿qué papel jugaron los ayuntamientos, cuál sería su función en los conflictos de tierras, sobre todo comunales y ejidales? El riesgo de perder la tierra era una amenaza para la economía de los pueblos indígenas; pero también lo era para la base de la cohesión identitaria. Esta era una buena razón para acudir a los juzgados.

\section{EL ÁMBITO DE LA JUSTICIA EN LOS AYUNTAMIENTOS}

Desde la Constitución de Cádiz se fijaron los asuntos en los cuales el ayuntamiento tenía el deber de intervenir. Básicamente se relacionaban con el cuidado material del pueblo, conservar el orden público y el bienestar social. (Guzmán Pérez, 2002 , p. 313$)^{36} \mathrm{Y}$ para conseguir estos objetivos era necesario que hubiera una buena administración de justicia, una de sus principales funciones.

36 En Chiapas, la Constitución del estado de Chiapas de 1858, en su artículo 63, definía las funciones de los ayuntamientos. 
Mientras que la información que nos ofrecen los documentos de los repositorios municipales para el caso de Chiapas es bastante escasa, los archivos judiciales se han convertido en una fuente de datos con un valor incuestionable. Bien como jueces o bien como partes, la dinámica de los ayuntamientos dejó su impronta en estos acervos, a los que debemos acudir para complementar la información necesaria para los estudios sobre esta institución. En ellos no sólo encontraremos información relativa a pleitos, conflictos y prácticas judiciales. Afortunadamente, el desarrollo de cada proceso va acompañado de otra información, como calidad étnica de los intervinientes, ubicaciones geográficas, datos sobre vecindad, relaciones sociales, información laboral..., entre otra, que es de gran utilidad para nuestras investigaciones.

La relación de los ayuntamientos con la administración de justicia se dio a través del juez conciliador, que no era otro que el alcalde electo en cada nuevo año en el momento de las elecciones de la corporación municipal. Como juzgado local, era el primer espacio en la resolución de conflictos, anterior al de primera instancia que se ubicaba en la cabecera de partido. En estos juzgados se dirimían los asuntos problemáticos y los pleitos del pueblo en juicios de conciliación y verbales. Sería largo enumerar las causas: pleitos por medidas de tierras, que fueron los más complejos; pero también casos comunes como, por ejemplo, el reparto de herencias, ${ }^{37}$ desavenencias que se producían en el ámbito familiar, social o laboral. Eran causas menores, tal como lo establecía la ley, y el objetivo era promover el acuerdo y la conciliación de las partes. En los distintos casos podemos comprobar que los indígenas tuvieron que adaptarse a las políticas y los procedimientos que imponía el derecho nacional. Por lo tanto, en asuntos de justicia ¿cómo y en qué dirección actuó el ayuntamiento constitucional a la hora de los conflictos? ¿Cómo podía la población indígena hacer valer sus derechos en un contexto ideológico liberal? ¿qué instrumentos utilizaba? ¿Podía recurrir a instituciones, como los ayuntamientos? ¿y si este era ladino? ¿Qué mecanismos usó la población que no se sentía representada para defender sus intereses?

Además de los juzgados locales, pensamos que había posibilidad de que los indígenas acudieran a sus propias formas de hacer justicia, lo que actualmente se conoce como usos y costumbres o derecho consuetudinario. Esto nos hace pensar en la existencia de una estructura de poder paralela a la constitucional, que no era reconocida legalmente, excepto en algunos periodos en que fue regulada por la legislación, pero que tenía una gran autoridad moral entre sus gentes. Desafortunadamente, la información disponible no nos permite profundizar en este tema. ${ }^{38}$

De cualquier forma, la relación entre ayuntamientos y administración de justicia es muy estrecha por lo que es importante profundizar en este sentido, a través de un análisis de los casos judiciales, para comprender y valorar si a los indígenas se les

37 AHE-FCG, expediente 162.

38 Véase Esquit (2002) para el caso de Tecpán, Guatemala. 
aplicaba la recta e imparcial justicia que el derecho positivo y los discursos del Estado proclamaban. Incluso hay que valorar si había condiciones para que estos acudieran a los juzgados, individual o colectivamente, para solicitar ser desagraviados o reclamar sus derechos. También debemos considerar las condiciones ideológicas que envolvían a los juzgados. Es claro que había una identificación entre determinados delitos y la población indígena, como, por ejemplo, vagancia y embriaguez. Ambas estaban condicionadas por la situación económica; pero además es importante considerar quien calificaba estas causas. En estos casos, lo hacían los alcaldes o los jueces de paz que por lo general eran ladinos.

También debemos reflexionar sobre cómo los indígenas se "movieron" en los juzgados, no sólo locales sino también otros superiores. Hay varios trabajos en los que se muestra que utilizaron sabiamente el derecho positivo para defenderse (Roseberry, 2004; Palomo, 2007), de forma especial cuando los intereses colectivos estaban en juego. Así sucedió sobre todo cuando los recursos naturales comunitarios eran los que peligraban.

En algunos pleitos el ayuntamiento dejó de ser juez para convertirse en parte de los conflictos. Analizaremos un caso relacionado con las tierras. La legislación del siglo XIX fomentó la desaparición de la propiedad comunal a favor de la individual (Cortés Máximo, 2003, p. 442) lo que sucedió de forma paulatina a lo largo del siglo. Según la ideología del momento, los recursos colectivos eran un lastre para el desarrollo y sólo la propiedad privada podría sacar a los indios de su apatía en cuanto a la producción.

En un principio se protegió a los ejidos coloniales. ${ }^{39}$ En diferentes decretos de las décadas de los veinte a los cuarenta, se estableció que los ayuntamientos eligieran y midieran sus dos porciones de ejido de acuerdo con su población, antes de cualquier medida de particulares en la zona ${ }^{40}$ Sin embargo esto último no siempre se cumplió y lo común fue la privatización temprana de tierras en manos de particulares. Esto provocó pleitos y juicios por el mejor derecho a los recursos, en los cuales los ayuntamientos tuvieron un papel protagónico.

En los archivos judiciales podemos encontrar estos pleitos por tierras. De su análisis se deduce que con frecuencia se cuestionaba la personalidad jurídica de los ayuntamientos como representantes de los pueblos en estos juicios; sin embargo, en varias ocasiones la corporación municipal aparece como parte defensora de los intereses comunes de los vecinos.

39 Sobre la conformación de los ejidos en Chiapas, puede consultarse el trabajo de Pedrero Nieto (2005)

40 Gobierno del Estado de Chiapas, Colección de leyes agrarias y demás disposiciones que se han emitido con relación al Ramo de Tierras, Imprenta Joaquín Armendáriz, sin lugar, 1878, citado por Fenner (2007, p. 35, nota 2). 
El 20 de mayo de 1861, el procurador síndico de Zapaluta, cuya población era en esas fechas mayoritariamente indígena, solicitó la segunda parte del ejido para el pueblo que, según la ley, le correspondía. Además, pedía que éste estuviera cerca de una montaña por "estar inmediato al pueblo y a la primera porción del ejido de éste", que colindaba con "la hacienda Santa Rita y San Isidro de cuyos dueños recibe ese vecindario ... muchos perjuicios" En la medición surgieron los desacuerdos con el C. Gregorio Culebro, dueño de una propiedad que lindaba con los terrenos nacionales de donde se había de conceder la segunda parte del ejido. Fue el ayuntamiento de Zapaluta el que entabló un largo pleito contra el señor Culebro por un desacuerdo de "seis caballerías y pico...", sobre las que él alegaba propiedad; hecho que finalmente no pudo comprobar. Tras un largo pleito el juzgado falló a favor del pueblo de Zapaluta quien pudo seguir con el aprovechamiento del terrero.

Otro asunto en el que intervino el cuerpo municipal fue en los problemas ocasionados en las relaciones laborales, concretamente con la mano de obra indígena para diferentes actividades productivas o de servicio. Los ayuntamientos de los pueblos de Chiapas tuvieron que organizar el flujo de mano de obra hacia las grandes fincas de café (Rus, 2005) de la misma forma que sucedió en algunos departamentos de Guatemala, en donde la municipalidad indígena tenía como propósito aliviar en la medida de lo posible la presión que sentían los indígenas por motivo de la demanda de mano de obra (Esquit, 2002). En este sentido, los ayuntamientos también actuaron de "intermediarios" en la resolución de conflictos que surgían entre los "sirvientes" y sus amos o con otras personas, para evitar los abusos. Generalmente, estos problemas se solucionaban en los juicios de conciliación.

\section{GONGLUSIONES}

Lo que contiene este texto son solo algunos de los temas y perspectivas con las que podemos acercarnos al estudio de los ayuntamientos decimonónicos en Chiapas. No se limita al estudio institucional y su funcionamiento; con esta propuesta pretendo ir un paso más allá, para ofrecer líneas de análisis y comprensión acerca del poder local desde la historia política y social. Esto implica adentrarse en el mundo de relaciones, coyunturas y circunstancias que rodean las dinámicas que se produjeron durante el siglo XIX entre la población indígena, el Estado y los demás grupos de población; en Chiapas, particularmente los ladinos. Por ello son importantes realizar estos estudios desde los actores sociales y la vida cotidiana de los pueblos.

Esta institución fue una de las más cercanas a la población; por lo tanto, su análisis es imprescindible para conocer la historia desde abajo, desde la conformación y las transformaciones que tuvieron los pueblos indígenas. Se propone el abordaje conceptual que envuelve todos los procesos desarrollados. También se deben incluir las particularidades locales y regionales que estuvieron presentes en la creación de los ayuntamientos, ya que este contexto nos explica las características institucionales y 
también sociales de las corporaciones municipales;nos permitenconocerla importancia de la participación de los diferentes grupos sociales en la vida política local.Asimismo, es necesario abordar los distintos ámbitos en los que intervenían los ayuntamientos, particularmente el económico y el relacionado con la administración de justicia.

Son asuntos pendientes, algunos de los cuales, como he señalado, han sido abordados con anterioridad por otras investigaciones; pero considero que es importante realizar un análisis en conjunto, que contenga no sólo la caracterización de la institución sino también, dentro de lo posible, las particularidades regionales y variantes temporales.

Soy consciente que esta es sólo una propuesta; que ni es la única perspectiva, ni están todos los temas que rodean al poder local. Pero este texto puede servir para abrir una discusión, o más bien continuarla, pues ya hay una cierta tradición historiográfica sobre este tema. Posiblemente, muchas de las preguntas y perspectivas no son ni novedosas, pero considero que el conjunto de ellas pueden ser un aporte importante para la reflexión.

Esta propuesta se dirige, finalmente, a reflexionar sobre el papel y la posición de los pueblos indígenas frente al Estado y las élites sociales. Esto nos permite poner sobre la mesa múltiples matices sobre las dinámicas cotidianas que se desarrollaban en el ámbito local. No debemos pensar que toda la población indígena se veía favorecida ni participaba en estos procesos políticos; también aquí podemos hablar de una "élite" indígena que actuaba en estos espacios de representación. Pero el análisis desde la perspectiva de la participación de autoridades indígenas en los espacios de poder local nos puede cambiar la idea tradicional sobre el lugar que ocupó esta población en los procesos históricos relacionados con el gobierno, el poder y la autoridad; y en general, puede posicionarlos y darles un papel mucho más activo y definitorio que lo que la historiografía les ha dado.

\section{BIBLIOGRAFÍA}

Acevedo Rodrigo, Ariadna y López Caballero, Paula (2012) Ciudadanos inesperados: espacios para la formación de la ciudadanía ayer y hoy. México: El Colegio de México.

Alda Mejías, Sonia (2000). La participación indígena en la construcción de la república de Guatemala, S. XIX. Colección de Estudios, núm. 69. Madrid: Universidad Autónoma de Madrid.

Annino, Antonio (1995). Historia de las elecciones en Iberoamérica, siglo XIX. México: Fondo de Cultura Económica.

Annino, Antonio (1995). Cádiz y la revolución territorial de los pueblos mexicanos 18121821. En Annino, Antonio (coord.), Historia de las elecciones en Iberoamérica, siglo XIX. México: Fondo de Cultura Económica, pp. 177-226. 
Annino, Antonio (2002). El Jano bifronte. En Reina, Leticia y Servín, Elisa (coords.), Crisis, Reforma y Revolución. México: historias de fin de siglo. México: Taurus, Consejo Nacional para la Cultura y las Artes, Instituto Nacional de Antropología e Historia, pp. 209-251.

Annino, Antonio (2003). Soberanías en lucha. En Annino, Antonio (coord.), Inventando la Nación: Iberoamérica siglo XIX. México: Fondo de Cultura Económica, pp. 152-184.

Avendaño, Xiomara (1997). Pueblos indígenas y república en Guatemala, 1812-1870. En Reina, Leticia (coord.). La reindianización de América, siglo XIX, México: Siglo XXI, CIESAS, pp. 109-120.

Ávila, Alfredo (2007). Liberalismos decimonónicos: de la historia de las ideas a la historia cultural e intelectual. En Palacios, Guillermo (coord.), Ensayos sobre la nueva historia política de América Latina, siglo XIX. México: El Colegio de México, pp. 111-146.

Barrera Aguilera, Oscar (2017). Las Terrazas de Los Altos: lengua, tierra y población en la Depresión Central de Chiapas. Tesis de doctorado. México: Centro de Estudios Históricos, El Colegio de México.

Barrios, Lina (1998). La alcaldía indígena en Guatemala: de 1821 a la revolución de 1945. Guatemala: Universidad Rafael Landívar.

Borrero Vega, Ana Luz (2014). El Legado de Cádiz: ciudadanía y cultura política en la Gobernación de Cuenca, 1812-1814. Procesos: Revista Ecuatoriana de Historia, [en línea] núm. 39, 9-36. DOI: https://doi.org/10.29078/rp.v1i39.79

Benjamin, Thomas Louis (1990). El camino a Leviatán: Chiapas y el Estado mexicano, 1891-1947. México: Consejo Nacional para la Cultura y las Artes.

Burguete Cal y Mayor, Araceli (2011). Municipalización del gobierno indígena e indianización del gobierno municipal en América Latina. Revista Pueblos y fronteras digital [en línea] vol. 6, n. 11, pp. 38-88. DOI: https://doi.org/10.22201/ cimsur.18704115e.2011.11.134

Burguete Cal y Mayor, Ruby Aracely (2016). Remunicipalización y luchas por la creación de nuevos municipios en México en la coyuntura zapatista (1994-2010). Tesis de doctorado. México: Universidad Nacional Autónoma de México.

Buve, Raymond (2007). Una historia particular. Tlaxcala en el proceso del establecimiento de la primera república federal. En Ortiz Escamilla, Juan y Serrano Ortega, José Antonio. Ayuntamientos y liberalismo gaditano en México. México: El Colegio de Michoacán, Universidad Veracruzana, pp. 55-88.

Cámara Barbachano, Fernando (1966). Persistencia y cambio cultural entre tzeltales de los altos de Chiapas: estudio comparativo de las instituciones religiosas y políticas de los municipios de Tenejapa y Oxchuc. México: Escuela Nacional de Antropología e Historia. 
Cancian, Frank (1976). Economía y prestigio en una sociedad maya. El sistema religioso de cargos en Zinacantan. México: Instituto Nacional Indigenista.

Cortés Máximo, Juan Carlos (2003). La comunidad de Tarímbaro. Gobierno indígena, arrendamiento y reparto de tierras, 1822-1884. En Paredes Martínez, Carlos y Terán, Marta (coords.). Autoridad y gobierno indígena en Michoacán (2 tomos). Zamora: El Colegio de Michoacán, Centro de Investigaciones y Estudios Superiores en Antropología Social, Instituto Nacional de Antropología e Historia, Universidad Michoacana.

Cortés Máximo, Juan Carlos (2012). De repúblicas de indios a ayuntamientos constitucionales: pueblos sujetos y cabeceras de Michoacán, 1740-1831. Morelia, Mich.: Universidad Michoacana de San Nicolás de Hidalgo, Instituto de Investigaciones Históricas.

Dublán, Manuel y José María Lozano (1876). Legislación mexicana o colección completa de las disposiciones legislativas expedidas desde la Independencia de la República. [en línea]. Disponible en: http://biblioweb.dgsca.unam.mx/ dublanylozano/ Consultado en 2008.

Escobar Ohmstede, Antonio (1996). Del gobierno indígena al Ayuntamientos constitucional en las Huastecas hidalguense y veracruzana, 1780-1853. Mexican Studies/Estudios Mexicanos, vol. 12, núm. 1.

Escobar Ohmstede, Antonio (1997). Los ayuntamientos y los pueblos indios en la sierra huasteca: conflictos entre nuevos y viejos actores, 1812-1840. En Reina, Leticia. La reindianización de América, Siglo XIX. México: Siglo XXI, pp. 294-316.

Escobar Ohmstede, Antonio (2010). ¿Qué tipo de naciones percibimos en la América Latina del siglo XIX? Sociedade e Cultura. Revista de Pesquisas e Debates em Ciéncias Sociales [en línea], vol. 13, núm. 1, enero-julio, pp. 39-53. Disponible en: https://www.redalyc.org/articulo.oa?id=70315011005. Consultado el 13 de enero de 2020.

Escobar Ohmstede, Antonio et al. (2010). La arquitectura histórica del poder. Naciones, nacionalismos y estados en América Latina. Siglos XVIII, XIX y XX. México: El Colegio de México, Centro de Estudios para el Desarrollo Laboral y Agrario.

Escobar Ohmstede, Antonio et al. (2015). Los efectos del liberalismo en México: Siglo $X I X$. Hermosillo, Son.: El Colegio de Sonora, Centro de Investigaciones y Estudios Superiores en Antropología Social.

Esquit, Edgar (2002). Otros poderes, nuevos desafios. Relaciones interétnicas en Técpan y su entorno departamental (1871-1935). Guatemala: Instituto de Estudios Interétnicos.

Fenner, Justus (2007). Pérdida o permanencia: el acaparamiento de las tierras colectivas en Chiapas durante el porfiriato. Un acercamiento a la problemática desde los expedientes del juzgado de distrito (1910-1876). Revista Pueblos y frontera digital [en línea] núm. 3. Disponible en: http://www.pueblosyfronteras.unam.mx. Consultado el 10 de junio de 2015. 
Galante, Mirian (2004). El liberalismo en la historiografía mexicanista de los últimos veinte años, Secuencia [en línea], núm. 58, pp. 161-188. DOI: https://doi. org/10.18234/secuencia.v0i58.843

Gayol, Víctor (2012). Formas de gobierno en México: poder político y actores sociales a través del tiempo. Zamora, Mich.: El Colegio de Michoacán.

Guarisco Canseco, Claudia (2003). Los indios del valle de México y la construcción de una nueva sociabilidad política. 1770-1835. Zinacantepec, Estado de México: El Colegio Mexiquense A.C.

Guarisco, Claudia (2019). Modernidad política y pueblos de indios en México, 17701856 (1 de 3). El Presente del Pasado [en línea], 14 de octubre de 2019. Disponible en: https://elpresentedelpasado.com/2019/10/14/modernidad-politica-y-pueblosde-indios-en-mexico-1770-1856-1-de-3/ Consultado el 11 de julio de 2020.

Güemez Pineda, Arturo (2005). Mayas. Gobierno y tierras frente a la acometida liberal en Yucatán, 1812-1847. Zamora, Michoacán: Colegio de Michoacán, Universidad Autónoma de Yucatán.

Guerra, François-Xavier (1993). Modernidad e independencias. Ensayos sobre las revoluciones hispánicas. México: Fondo de Cultura Económica.

Guerra, François-Xavier (1994). La desintegración de la monarquía hispánica: revolución e independencias. En: Annino, Antonio et al. De los imperios a las naciones. Iberoamérica. Zaragoza: IberCaja, pp. 195-227.

Guerra, François-Xavier (1995). México: del Antiguo Régimen a la Revolución. México: Fondo de Cultura Económica.

Guerrero, Andrés (2010). Administración de poblaciones, ventriloquía y transescritura: análisis históricos, estudios teóricos. Quito y Lima: Facultad Latinoamericana de Ciencias Sociales e Instituto de Estudios Peruanos.

Gutiérrez Cruz, Sergio Nicolás y Parrilla Albuerne, Ana María (2018). Alcaldes y subdelegados de la Intendencia de Ciudad Real de Chiapa: autoridades enfrentadas en vísperas de la Independencia. Revista Pueblos y fronteras digital [en línea], vol. 13, e-306, pp. 1-27. DOI: https://doi.org/10.22201/cimsur.18704115e.2018.25.306

Guzmán Pérez, Moisés (2002). Cádiz y el ayuntamiento constitucional en los pueblos indígenas de la Nueva España, 1820-1825. En De súbditos del rey a ciudadanos de la nación. Castellón, Universidad Jaume I.

Herrera Mena, Sajid Alfredo (2013). El ejercicio de gobernar: del cabildo borbónico al ayuntamiento liberal. El Salvador colonial, 1750-1821. Castellón: Universitat Jaume I.

Instituto Nacional de Antropología e Historia (2007). Estado que manifiesta los pueblos donde hay ayuntamientos constitucionales en virtud del artículo 75 de la constitución del Estado y ley provisional de 30 de noviembre de 825 con expresión de las cabezas de partido a que pertenecen. 1827. En Catálogo electrónico, Archivo Histórico de Chiapas, Biblioteca Manuel Orozco y Berra. México: Apoyo al Desarrollo de Archivos y Bibliotecas de México. 
Irurozqui, Marta (2004). La ciudadanía en debate en América Latina. Discusiones historiográficas y una propuesta teórica sobre el valor público de la infracción electoral. [en línea], Lima: Instituto de Estudios Peruanos. Disponible en: http:// lanic.utexas.edu/project/laoap/iep/ddt139.pdf. Consultado el 16 de septiembre de 2013.

Lempérière, Annick (2007). La historiografía del estado en Hispanoamérica. Algunas reflexiones. En Palacios, Guillermo (coord.). Ensayos sobre la nueva historia política de América Latina, siglo XIX. México: El Colegio de México, pp. 45-62.

MacLeod, Murdo J. (1983). Papel social y económico de las cofradías indígenas de la Colonia en Chiapas. Mesoamérica, núm. 5, pp. 64-86.

Machuca Gallegos, Laura (2014). Ayuntamientos y sociedad en el tránsito de la época colonial al siglo XIX. Reinos de Nueva España y Guatemala. México: Centro de Investigaciones y Estudios Superiores en Antropología Social.

Marino, Daniela (2006). La modernidad a juicio: los pueblos de Huixquilucan en la transición jurídica (Estado de México, 1856-1911). Tesis de doctorado. México: El Colegio de México.

Mendoza García, Edgar (2011). Del cabildo colonial a la municipalidad republicana: territorio del gobierno local en Oaxaca. En Salinas Sandoval, María del Carmen, Birrichaga Gardida, Diana y Escobar Ohmstede, Antonio (coords.). Poder y gobierno local en México. 1808-1857. Zinacantepec, Edo. de México: El Colegio Mexiquense, El Colegio de Michoacán, Universidad Autónoma del Estado de México, pp. 375-409.

Obara-Saeki, Tadashi (2010). Ladinización sin mestizaje: historia demográfica del área chiapaneca, 1748-1813. Tuxtla Gutiérrez, Chis.: Consejo Estatal para las Culturas y las Artes de Chiapas.

Ortiz Escamilla, Juan y Serrano Ortega, José Antonio (2007). Ayuntamientos y liberalismo gaditano en México. Zamora, Mich.: El Colegio de Michoacán, Universidad Veracruzana.

Ortiz Herrera, Rocío (2003). Pueblos indios, iglesia católica y élites políticas en Chiapas (1824-1901). Una perspectiva comparativa. Tuxtla Gutiérrez, Chis.: Consejo Estatal para la Cultura y las Artes de Chiapas.

Ortiz Herrera, Rocío (2018a). Ayuntamientos chiapanecos: fiscalidad, elecciones, ciudadania y defensa de bienes de comunidad desde la colonia hasta el inicio de la Revolución en Chiapas. Tuxtla Gutiérrez, Chis.: Universidad de Ciencias y Artes de Chiapas, El Colegio de Michoacán.

Ortiz Herrera, Rocío (2018b). Voto indígena, ayuntamientos y formación del estado en Chiapas, 1904-1917. En Ortiz Herrera, Rocío, Ayuntamientos chiapanecos: fiscalidad, elecciones, ciudadanía y defensa de bienes de comunidad desde la colonia hasta el inicio de la Revolución en Chiapas. Tuxtla Gutiérrez, Chis.: Universidad de Ciencias y Artes de Chiapas, El Colegio de Michoacán. 
Palomeque Torres, Silvia (2000). La ciudadanía y el sistema de gobierno en los pueblos de Cuenca (Ecuador). En König, Hans Joachim et al. Estado-nación, Comunidad Indígena, Industria. Tres debates al final del Milenio, Cuadernos de Historia Latinoamericana. AHILA, no 8. Ridderkerk: Asociación de Historiadores Latinoamericanistas Europeos, pp. 115-142.

Palomeque, Silvia (1992). Estado y comunidad en la región de Cuenca en el siglo XIX. Las autoridades indígenas y su relación con el Estado. En Bonilla, Heraclio (comp.) Los Andes en la encrucijada. Indios, comunidades y estado en el siglo XIX. Ecuador: Ed. Libri-Mundi, Facultad Latinoamericana de Ciencias Sociales, pp. 391-419.

Palomo Infante, María Dolores (2007). "Enredos y sutilezas del derecho" en defensa de los bienes comunes. La hacienda San Pedro Pedernal de Huixtán, Chiapas. 17901865. Revista Pueblos y Fronteras digital [en línea], núm. 3. DOI: https://doi. org/10.22201/cimsur.18704115e.2007.3.231

Palomo Infante, María Dolores (2009a). Juntos y congregados. Historia de las cofradías en los pueblos de indios tzotziles y tzeltales de Chiapas (siglos XVI al XIX). México: Centro de Investigaciones y Estudios Superiores en Antropología Social.

Palomo Infante, María Dolores (2009b). Los ayuntamientos de los pueblos indígenas de Chiapas en el siglo XIX y su relación con los asuntos de justicia. Anuario de Estudios Americanos, [en línea], Vol. 66, No. 1. DOI: https://doi.org/10.3989/ aeamer.2009.v66.i1.302

Palomo Infante, María Dolores (2015). Presencia indígena en la representación política de los pueblos de las regiones de Los Llanos y los Zendales, Chiapas, siglo XIX. En Reina, Leticia. Pueblos indígenas en Latinoamérica: Incorporación, conflicto, ciudadanía y representación. Siglo XIX. México: Instituto Nacional de Antropología e Historia, pp. 159-186.

Palomo Infante, María Dolores (2018). "De los ciudadanos chiapanecos". Ciudadanía y espacios políticos indígenas, 1826-1858. En Ortiz Herrera, Rocío (coord.). Ayuntamientos chiapanecos: fiscalidad, elecciones, ciudadanía y defensa de bienes de comunidad desde la colonia hasta el inicio de la Revolución en Chiapas. Tuxtla Gutiérrez, Chiapas: Universidad de Ciencias y Artes de Chiapas, El Colegio de Michoacán.

Palomo Infante, María Dolores (2020). Demografía y sociedad: registros de población y proceso de ladinización de los pueblos de los Llanos y Zendales de Chiapas (México), hasta 1877. Collection Publications du GRECUN. EA 369, Centre de Recherches Ibériques et Ibéro-américaines, Universidad Paris Nanterre, pp. 15-33.

Parrilla Albuerne, Ana María (2018). Propios y arbitrios del ayuntamiento de Ciudad Real. Un pulso entre el poder local y los nuevos funcionarios de la intendencia, 17861812. En Ortiz Herrera, Rocío (coord.). Ayuntamientos chiapanecos: fiscalidad, elecciones, ciudadanía y defensa de bienes de comunidad desde la colonia hasta el inicio de la Revolución en Chiapas. Tuxtla Gutiérrez, Chis.: Universidad de Ciencias y Artes de Chiapas, El Colegio de Michoacán. 
Pedrero Nieto, Gloria (2005). La evolución del ejido en Chiapas (siglo XIX). En Olivera, Mercedes y Palomo, María Dolores (coords.). Chiapas, de la Independencia a la Revolución. México: Centro de Investigaciones y Estudios Superiores en Antropología Social, Consejo Estatal de Ciencia y Tecnología.

Peralta, Víctor (1991). En pos del tributo en el Cusco rural, 1826-1854. Perú: Centro de Estudios Regionales Andinos "Bartolomé de las Casas".

Quijada Mauriño, Mónica et Al (2000). Homogeneidad y nación: con un estudio de caso: Argentina, siglos XIX y XX. Madrid: Consejo Superior de Investigaciones Científicas.

Rodas, Isabel (1995). Ladinos y pueblos de indios, el caso de Patzicía colonial. París: Universidad de París 8, Programa Guatemala, Guatemala.

Rodas, Isabel (1997). Élite ladina-vanguardia indígena de la intolerancia a la violencia Patzicía. Guatemala: Universidad de San Carlos de Guatemala.

Rodríguez Castillo, Luis (2003). Nuevos espacios de representación política en la selva fronteriza. La disputa por la remunicipalización en Chiapas, México. LiminaR Estudios Sociales Y Humanísticos [en línea], 1(2), 26-42. https://doi.org/10.29043/ liminar.v1i2.132

Roseberry, William (2004). "El estricto apego a la ley". Ley liberal y derecho comunal en el Pátzcuaro porfiriano. En Roth Seneff, Andrew. Recursos contenciosos. Ruralidady reformas liberales en México. Zamora, Mich.: El Colegio de Michoacán, pp. 43-84.

Rus, Jan, (2005). El café y la recolonización de los Altos de Chiapas, 1892-1910. En Olivera, Mercedes y Palomo, María Dolores. Chiapas, de la Independencia a la Revolución. México: Centro de Investigaciones y Estudios Superiores en Antropología Social, Consejo Estatal de Ciencia y Tecnología.

Rus, Jan y Wasserstrom, Robert (1980). Civil-religious hierarchies in central Chiapas: a critical perspective. American Ethnologist, 7, pp. 466-478.

Sábato, Hilda (1999). Ciudadanía política y formación de las naciones. Perspectivas históricas de América Latina. México: Fideicomiso Historia de las Américas, Fondo de Cultura Económica.

Salinas Sandoval, María del Carmen et al. (2011). Poder y gobierno local en México, 1808-1857. Toluca: El Colegio Mexiquense.

Sánchez Rafael, Miguel Ángel (2018). Reformas y finanzas en la esfera municipal. Chiapas, 1881-1915. En Ortiz Herrera, Rocío (coord.). Ayuntamientos chiapanecos: fiscalidad, elecciones, ciudadanía y defensa de bienes de comunidad desde la colonia hasta el inicio de la Revolución en Chiapas. Tuxtla Gutiérrez, Chis.: Universidad de Ciencias y Artes de Chiapas, El Colegio de Michoacán.

Thurner, Mark (1996). "De alcaldes y caudillos: posibilidad y problema nacional en la crisis peruana de fin de siglo". En Bonilla, Heraclio y Guerrero, Andrés (eds.). Los pueblos campesinos de las Américas. Etnicidad, cultura e historia en el siglo XIX. Colombia: Universidad Industrial de Santander, pp. 223-238. 
Torres Freyermuth, Amanda Úrsula (2017). Los hombres de bien. Un estudio de la elite politica en Chiapas (1824-1835). México: UNAM.

Vallejo Flores, Mercedes Verónica (2017).Justicemunicipale etjusticiables à Guadalajara (1821-1846). Fonctionnement et portée d'une institution de proximité dans une période de transition. Tesis de doctorado. Paris: Ecole Doctorale d'Historire (ED 113), Université Paris I-Panthéon-Sorbonne.

Vázquez Olivera, Mario (2010). "Un remedo de antiguos atenienses". Los ayuntamientos de Chiapas en la proclamación de la independencia y la unión a México. En Vázquez Olivera, Mario, Chiapas, años decisivos. Independencia, unión a México y Primera República Federal. Tuxtla Gutiérrez: UNICACH, pp. 67-92. 
